\title{
Influence of two-photon absorption on soliton self-frequency shift
}

\author{
Steffensen, Henrik; Agger, Christian; Bang, Ole
}

Published in:

Optical Society of America. Journal B: Optical Physics

Link to article, DOI:

10.1364/JOSAB.29.000484

Publication date:

2012

Link back to DTU Orbit

Citation (APA):

Steffensen, H., Agger, C., \& Bang, O. (2012). Influence of two-photon absorption on soliton self-frequency shift. Optical Society of America. Journal B: Optical Physics, 29(3), 484-492.

https://doi.org/10.1364/JOSAB.29.000484

\section{General rights}

Copyright and moral rights for the publications made accessible in the public portal are retained by the authors and/or other copyright owners and it is a condition of accessing publications that users recognise and abide by the legal requirements associated with these rights.

- Users may download and print one copy of any publication from the public portal for the purpose of private study or research.

- You may not further distribute the material or use it for any profit-making activity or commercial gain

- You may freely distribute the URL identifying the publication in the public portal

If you believe that this document breaches copyright please contact us providing details, and we will remove access to the work immediately and investigate your claim. 


\title{
Influence of two-photon absorption on soliton self-frequency shift
}

\author{
Henrik Steffensen, ${ }^{*}$ Christian Agger, and Ole Bang \\ DTU Fotonik, Technical University of Denmark, Ørsteds Plads, Building 343, DK-2800 Kongens Lyngby, Denmark \\ *Corresponding author: hste@fotonik.dtu.dk
}

Received October 6, 2011; revised December 6, 2011; accepted December 12, 2011; posted December 14, 2011 (Doc. ID 156064); published February 29, 2012

\begin{abstract}
In this paper we develop an analytical model for the soliton self-frequency shift, which includes second-and thirdorder dispersion, self-steepening, the full Raman term, and, for the first time to our best knowledge, the effect of two-photon absorption (TPA). We show that TPA can have a significant effect on soliton dynamics in soft-glass materials such as chalcogenides, by severely depleting a soliton and thereby limiting the achievable redshift. Based on the model, we derive a nonlinear loss length after which the redshift is effectively halted by TPA, which proves to be a useful design tool. (C) 2012 Optical Society of America
\end{abstract}

OCIS codes: $\quad 060.4370,060.5530$.

\section{INTRODUCTION}

Broadband light sources in the mid-infrared range, 2 to $15 \mu \mathrm{m}$, are of significant interest due to the many infrared appli-

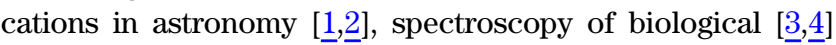
and chemical [5] samples, gas sensing [6], and military countermeasures [7]. Broadband light sources based on supercontinuum generation (SCG) [] ] in silica fibers are widely used in the visible and near-infrared wavelength range [9]. However, a mid-infrared supercontinuum light source cannot use silica fibers because of the loss edge at around $2.2 \mu \mathrm{m}$. Instead, other glasses with a higher transparency in the mid-infrared, such as ZBLAN [10,11], bismuth [12], tellurite [13,14], or chalcogenide $[\underline{15}, \underline{16}]$, can be used. Chalcogenide glasses generally have a nonlinear index 2 orders of magnitude higher than silica, whereas bismuth and tellurite have 1 order of magnitude higher, and finally ZBLAN has a nonlinear index comparable to that of silica [17]. The material zero dispersion wavelength (ZDW) is also higher than that of silica, with chalcogenide having the highest at around 4 to $5 \mu \mathrm{m}$, depending on the chemical composition [12].

The fundamentals in SCG are pumping the nonlinear fiber close to the ZDW and by modulation instability or soliton fission to generate fundamental solitons and have them redshift [8]. Soliton self-frequency shifting (SSFS) is thus essential in obtaining a broad mid-infrared supercontinuum spectrum. Various methods are used to reduce the ZDW of the fiber to enable the use of fiber lasers, such as using photonic crystal fibers (PCFs) $[\underline{8}, 18]$ that can be dispersion engineered for optimal SCG [19], tapers [15,20-22], or by the use of silica fiber to either redshift the pulse [23] or to initialize the SCG [10] before coupling to a soft-glass fiber.

The SSFS effect was analytically shown by Gordon [24] by looking at the Raman amplification as a perturbation to the nonlinear Schrödinger equation (NLS). Later works extended the description by including self-steepening and third-order dispersion (TOD) for long pulses [25] and linear losses [26]. Judge $e t a l$. extended the original model to be used in fiber tapers $[27,28]$, and recent work has been shown that includes the full Raman description as well as linear loss, TOD, and self-steepening effects by the use of the method of moments [29]. As chalcogenide is increasingly being used as a new material for SCG, it is now necessary to extend the previous work to also include two-photon absorption (TPA), which is present in various chalcogenide materials, for instance, $\mathrm{As}_{2} \mathrm{Se}_{3}$. As it was shown that including loss in the description of SSFS had a great effect of the predicted redshift [26], it is expected that TPA will further reduce the redshift.

\section{METHOD OF MOMENTS}

The pulse propagation in an optical fiber is modeled with the generalized nonlinear Schrödinger equation (GNLS) [29]

$$
\begin{aligned}
\partial_{z} u(z, t)+\frac{\alpha}{2} u(z, t)-i \sum_{m \geq 2} \frac{i^{m} \beta_{m}}{m !} \partial_{t}^{m} u(z, t) \\
=i \sum_{n \geq 0} \frac{i^{n} \gamma_{n}}{n !} \partial_{t}^{n}\left\{u(z, t) \int R\left(t^{\prime}\right)\left|u\left(z, t-t^{\prime}\right)\right|^{2} \mathrm{~d} t^{\prime}\right\},
\end{aligned}
$$

where $u$ is the complex field envelope at carrier frequency $\omega_{0}$; $\alpha$ is the linear loss; $\beta_{m}$ and $\gamma_{m}$ are the $m$ th derivative with respect to $\omega$ at $\omega_{0}$ of the propagation constant, $\beta(\omega)$ and the nonlinear coefficient, $\gamma(\omega)$, respectively; and finally $R(t)$ is the nonlinear time response given by $R(t)=\left(1-f_{R}\right) \delta(t)+$ $f_{R} h_{R}(t)$, which includes both the instantaneous Kerr response and the delayed Raman response, given as $h_{R}(t)$. The zeroth derivative is per definition the function itself, e.g., $\partial_{t}^{0} u=u$, and the integral is performed over the entire domain, e.g., $\int u \mathrm{~d} t=\int_{-\infty}^{\infty} u \mathrm{~d} t$. When TPA is present, the nonlinear coefficient, $\gamma_{0}$, is given by [30]

$$
\gamma_{0}=\frac{\omega_{0} n_{2}}{c A_{\mathrm{eff}}}+i \frac{\beta_{\mathrm{TPA}}}{2 A_{\mathrm{eff}}},
$$

where $n_{2}=n_{2}(\omega)$ is the nonlinear refractive index, $c$ is the speed of light, $\beta_{\mathrm{TPA}}=\beta_{\mathrm{TPA}}(\omega)$ is the TPA coefficient, and $A_{\text {eff }}=A_{\text {eff }}(\omega)$ is the effective mode area. The TPA constant is often expressed in terms of the figure of merit (FOM) defined as $\mathrm{FOM}=n_{2} /\left(\lambda \beta_{\mathrm{TPA}}\right)[\underline{31}]$. 
For this investigation, we include the first two dispersion terms, namely, the group velocity dispersion, $\beta_{2}$, and TOD, $\beta_{3}$, terms, as well as the first two nonlinear terms, $\gamma_{0}$ and $\gamma_{1}$. The first nonlinear term includes the Kerr nonlinearity, the Raman effect, and the TPA, whereas the second contains the self-steepening and the first-order frequency variation of the Kerr nonlinearity and the TPA constant.

The work in this paper is based on utilizing the method of moments $[32,25]$. The more common variational technique cannot be applied because the GNLS equation, Eq. (1), does not have a Lagrangian when the temporally asymmetric nonlocal Raman term is included. Had the nonlocality been symmetric, then variational techniques could have been applied $[33,34]$. The method of moments is based upon defining an ansatz function together with a set of moments. The simplest approximation to the GNLS equation is the NLS equation, which has an analytic solution in the case of anomalous dispersion in the form of a soliton. The ansatz will therefore be based on a fundamental soliton and described as

$$
\begin{aligned}
u(z, t)= & \sqrt{P(z)} \operatorname{sech}\left(\frac{t-t_{c}(z)}{T(z)}\right) \\
& \times \exp \left[i \Phi(z)-i b(z)\left(t-t_{c}(z)\right)-i \mu(z)\left(t-t_{c}(z)\right)^{2}\right],
\end{aligned}
$$

where $P, t_{c}$, and $T$ are the peak power, the temporal shift, and the temporal width, respectively; $b$ is the frequency shift; $\mu$ is the quadratic chirp; and $\Phi$ is the phase shift.

To determine the evolution of these parameters, we apply the method of moments to the problem. This is based on defining five moments given as

$$
\begin{aligned}
Q & =\int|u|^{2} \mathrm{~d} t \\
P_{M} & =\frac{1}{2} \int\left(u \partial_{t} u^{*}-u^{*} \partial_{t} u\right) \mathrm{d} t, \\
I_{1} & =\int t|u|^{2} \mathrm{~d} t, \\
I_{2} & =\int\left(t-t_{c}\right)^{2}|u|^{2} \mathrm{~d} t, \\
I_{3} & =\int\left(t-t_{c}\right)\left(u^{*} \partial_{t} u-u \partial_{t} u^{*}\right) \mathrm{d} t,
\end{aligned}
$$

which are necessary to describe the evolution of $P, t_{c}, T, b$, and $\mu$. It is also possible to describe the evolution of the phase shift by applying a sixth moment [35]. This is not done in this work as the evolution of the frequency is not coupled to the phase shift.

With the use of the ansatz given in Eq. (3), these moments can be evaluated and expressed as functions of the five pulse characteristics. These expressions are then differentiated with respect to $z$ and rewritten, which results in five differential equations, one for each of the pulse characteristics and all as a function of the derivatives of the moments. The derivatives of the moments can then also be evaluated by differentiating Eq. (4) and inserting Eqs. (1) and (3). The end result is five nonlinear differential equations for the pulse characteristics $P, t_{c}, T, b$, and $\mu$, which are rather long and provide no significant insight by themselves, so these can be found in Appendix A. This set of differential equations includes the full Raman term, and, as such, it includes integrals over the time response and $T$-dependent functions. However, for either very long or very short pulses, these integrals can be greatly reduced. For long pulses, the analytic model is reduced to

$$
\begin{gathered}
\partial_{z} b \approx-\frac{8}{15} \frac{\left(\gamma_{0}^{r}+b \gamma_{1}^{r}\right) P T_{R}}{T^{2}}+\frac{4}{3} \mu \gamma_{1}^{r} P-\frac{4}{3} \mu\left(\gamma_{0}^{i}+b \gamma_{1}^{i}\right) P T_{R} \\
-\frac{4}{5} \frac{\gamma_{1}^{i} P}{T^{2}}-4\left(\frac{\pi^{2}}{9}-\frac{2}{3}\right) \mu^{2} \gamma_{1}^{i} P T^{2}, \\
\partial_{z} t_{c} \approx b \beta_{2}+\frac{1}{2} \beta_{3}\left[\frac{\pi^{2}}{3} \mu^{2} T^{2}+\frac{1}{3 T^{2}}+b^{2}\right]+\gamma_{1}^{r} P \\
-\frac{2}{3}\left(\gamma_{0}^{i}+b \gamma_{1}^{i}\right) P T_{R}-2\left(\frac{\pi^{2}}{9}-\frac{2}{3}\right) \mu \gamma_{1}^{i} P T^{2}, \\
\partial_{z} T \approx 2 \mu T\left(\beta_{2}+b \beta_{3}\right)+\frac{8}{\pi^{2}} \frac{\gamma_{1}^{r} P T_{R}}{T}+\frac{4}{\pi^{2}}\left(\gamma_{0}^{i}+b \gamma_{1}^{i}\right) P T \\
-\left(\frac{4}{3}-\frac{12}{\pi^{2}}\right) \mu \gamma_{1}^{i} P T T_{R}, \\
\partial_{z} P \approx-\alpha P-2 \mu P\left(\beta_{2}+b \beta_{3}\right)-\left(\frac{8}{\pi^{2}}+\frac{8}{15}\right) \frac{\gamma_{1}^{r} P^{2} T_{R}}{T^{2}} \\
-\left(\frac{4}{3}+\frac{4}{\pi^{2}}\right)\left(\gamma_{0}^{i}+b \gamma_{1}^{i}\right) P^{2}-\frac{12}{\pi^{2}} \mu \gamma_{1}^{i} P^{2} T_{R}, \\
\partial_{z} \mu \approx\left(\frac{2}{\pi^{2}} \frac{1}{T^{4}}-2 \mu^{2}\right)\left(\beta_{2}+b \beta_{3}\right)+\frac{2}{\pi^{2}} \frac{\left(\gamma_{0}^{r}+b \gamma_{1}^{r}\right) P}{T^{2}} \\
-\left(\frac{8 \gamma_{1}^{r} P T_{R}}{T^{2}}-\frac{76}{15 \pi^{2}} \frac{\gamma_{1}^{i} P T_{R}}{T^{4}},\right.
\end{gathered}
$$

where $\gamma_{n}^{r}$ and $\gamma_{n}^{i}$ are the real and imaginary parts of $\gamma_{n}$, respectively, and

$$
T_{R}=f_{R} \int t h_{R}(t) \mathrm{d} t,
$$

which for silica, with a single resonance, has a value of approximately 3.2 fs and for ZBLAN, with two resonances, is approximately $0.15 \mathrm{fs}$ [36].

It was proposed by Chen et al. [29] to introduce a moving frequency frame, because the parameters $\beta_{2}$ and $\gamma_{0}$ appear in Eq. (5) as a linear expansion. The moving frequency frame is based on using a soliton with a fixed carrier frequency $\omega_{0}$ with the dispersion and nonlinearity parameters evaluated at this frequency and to update the frequency to follow the SSFS as $\omega_{0}(z+d z)=\omega_{0}(z)+d b$. With this transformation, Eq. ( $\left.\underline{5}\right)$ reduces to

$$
\begin{aligned}
\partial_{z} b \approx & -\frac{8}{15} \frac{\gamma_{0}^{r} P T_{R}}{T^{2}}+\frac{4}{3} \mu \gamma_{1}^{r} P-\frac{4}{3} \mu \gamma_{0}^{i} P T_{R}-\frac{4}{5} \frac{\gamma_{1}^{i} P}{T^{2}} \\
& -4\left(\frac{\pi^{2}}{9}-\frac{2}{3}\right) \mu^{2} \gamma_{1}^{i} P T^{2}, \\
\partial_{z} t_{c} \approx & \frac{1}{2} \beta_{3}\left[\frac{\pi^{2}}{3} \mu^{2} T^{2}+\frac{1}{3 T^{2}}\right]+\gamma_{1}^{r} P-\frac{2}{3} \gamma_{0}^{i} P T_{R} \\
& -2\left(\frac{\pi^{2}}{9}-\frac{2}{3}\right) \mu \gamma_{1}^{i} P T^{2},
\end{aligned}
$$




$$
\begin{gathered}
\partial_{z} T \approx 2 \mu T \beta_{2}+\frac{8}{\pi^{2}} \frac{\gamma_{1}^{r} P T_{R}}{T}+\frac{4}{\pi^{2}} \gamma_{0}^{i} P T-\left(\frac{4}{3}-\frac{12}{\pi^{2}}\right) \mu \gamma_{1}^{i} P T T_{R}, \\
\partial_{z} P \approx-\alpha P-2 \mu P \beta_{2}-\left(\frac{8}{\pi^{2}}+\frac{8}{15}\right) \frac{\gamma_{1}^{r} P^{2} T_{R}}{T^{2}} \\
-\left(\frac{4}{3}+\frac{4}{\pi^{2}}\right) \gamma_{0}^{i} P^{2}-\frac{12}{\pi^{2}} \mu \gamma_{1}^{i} P^{2} T_{R}, \\
\partial_{z} \mu \approx\left(\frac{2}{\pi^{2}} \frac{1}{T^{4}}-2 \mu^{2}\right) \beta_{2}+\frac{2}{\pi^{2}} \frac{\gamma_{0}^{r} P}{T^{2}}-\left(\frac{8}{15}-\frac{4}{\pi^{2}}\right) \frac{\mu \gamma_{1}^{r} P T_{R}}{T^{2}} \\
-\frac{76}{15 \pi^{2}} \frac{\gamma_{1}^{i} P T_{R}}{T^{4}} .
\end{gathered}
$$

The method of moments allows for the fiber parameters to be $z$ dependent, and, as such, the proposed model can also be used to investigate the effects of fiber tapers.

If we assume a wide and chirp-free pulse, while also considering the dispersion of the TPA to be negligible, i.e., $\gamma_{1}^{i}=0$, the differential equations for $P, T$, and $b$ can be reduced to

$$
\begin{gathered}
\partial_{z} P=-\alpha P-\left(\frac{4}{3}+\frac{4}{\pi^{2}}\right) \gamma_{0}^{i} P^{2}, \\
\partial_{z} T=\frac{8}{\pi^{2}} \frac{\gamma_{1}^{r} P T_{R}}{T}+\frac{4}{\pi^{2}} \gamma_{0}^{i} P T, \\
\partial_{z} b=-\frac{8}{15} \frac{\gamma_{0}^{r} P T_{R}}{T^{2}} .
\end{gathered}
$$

This set of reduced equations offers an insight into the effect of TPA. First, Eq. (8a) shows the model accurately includes the TPA effect as nonlinear power reducing term, as the sign of the second term on the right-hand side (RHS) is negative, as is the case for the linear loss term. From the second term in the RHS of Eq. (8b), it is additionally shown that the TPA initially increase the rate of pulse lengthening. The combined effect of decreased pulse power and an increased pulse width will, as is shown from Eq. (8c), reduce the rate of the SSFS.

The approximation, Eq. (8a), is analytically solvable and is used to define a nonlinear loss length, including both linear and TPA loss, as the length after which the peak power has been reduced to $1 / e$ of the initial value, which is given by

$$
L_{\mathrm{Loss}}=\frac{1}{\alpha} \ln \left[\frac{e+\left(\frac{4}{3}+\frac{4}{\pi^{2}}\right) \frac{\gamma_{0}^{i} P_{0}}{\alpha}}{1+\left(\frac{4}{3}+\frac{4}{\pi^{2}}\right) \frac{\gamma_{0}^{i} P_{0}}{\alpha}}\right] \text {, }
$$

where $P_{0}=P(0)$. This nonlinear loss length gives us a qualitative expression for the length after which the peak power is reduced to the extend where the redshifting of the soliton is effectively halted. For a case where TPA can be neglected, it is seen that the loss length is reduced to only depend on the linear loss.

\section{COMPARISON OF MODELS}

Let us first check our model in the simpler case where TPA is not included. We compare our full model, Eq. (A1) to simulations of Eq. (1), using the first two dispersion terms as well as the first two nonlinear terms as with the derivation of our model, and to the model proposed by Chen et al. [29].
We consider a fundamental soliton input at $1550 \mathrm{~nm}$ in a $50 \mathrm{~m}$ silica fiber with a loss of $0.2 \mathrm{~dB} / \mathrm{km}$. The dispersion parameters are assumed as $\beta_{2}=-5.1 \mathrm{ps}^{2} / \mathrm{km}$ and $\beta_{3}=$ $0.1 \mathrm{ps}^{3} / \mathrm{km}$. As we look at a case where TPA is neglected, the imaginary part of the nonlinear coefficients are all zero, and the real parts are assumed to be $\gamma_{0}^{r}=2 \mathrm{~W}^{-1} \mathrm{~km}^{-1}$ and $\gamma_{1}=\gamma_{0}^{r} / \omega_{0}$. The input peak power is defined by the chosen pulse width, according to the relation $P=\left|\beta_{2}\right| /\left(\gamma_{0}^{r} T^{2}\right)$, as we assume a fundamental soliton.

Figure 1 shows the results for two different soliton widths, $T=25 \mathrm{fs}$ and $T=100 \mathrm{fs}$. As seen, for the long pulse in Fig. 1(a), there is a good level of agreement between the variation of the center wavelength and the peak power for the full simulation of Eq. (1) (dashed), our model (solid), and the method of Chen et al. (dashed-dotted). The two models are nearly identical in their predictions. In contrast, for the shorter pulse in Fig. 1(b), there begins to be a greater difference between our model (solid) and that of Chen et al. (dashed-dotted); however, both models still have a good level
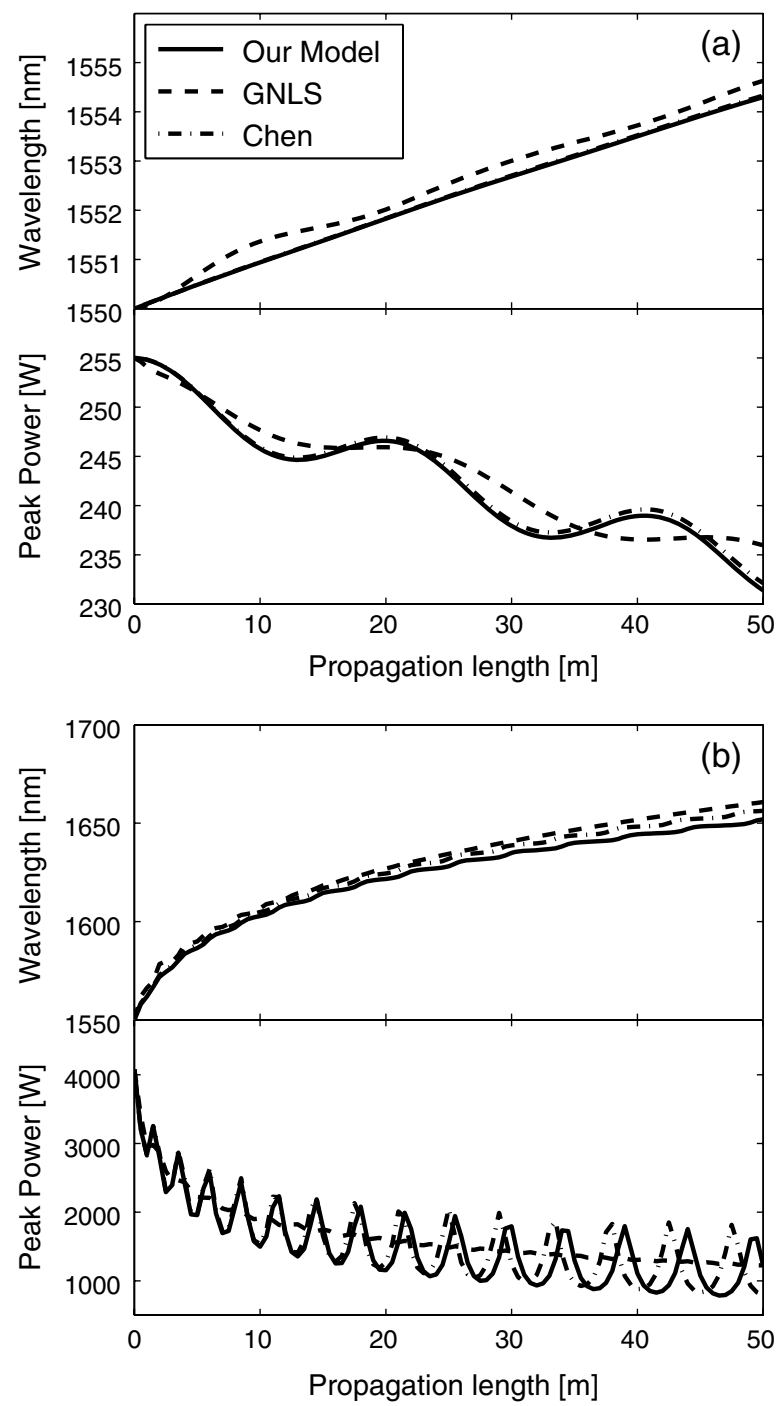

Fig. 1. Center wavelength (top) and peak power (bottom) as a function of propagation distance for (a) $T=100 \mathrm{fs}$ and (b) $T=25 \mathrm{fs}$ pulses. It shows that there is a good agreement between our model (solid) and the full simulation of Eq. (1) (dashed) and the model of Chen et al. (dashed-dotted). 


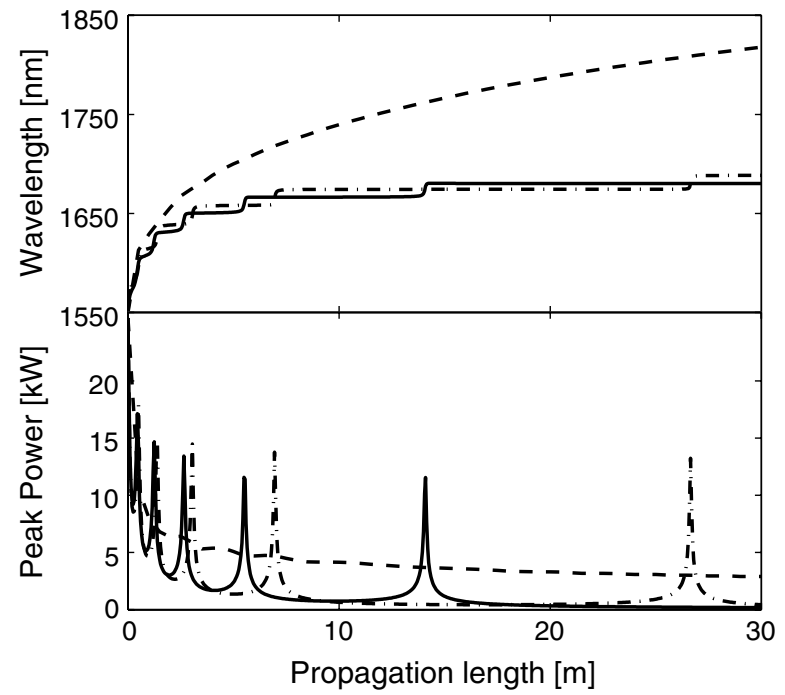

Fig. 2. Center wavelength (top) and peak power (bottom) as a function of propagation distance for a $T=10 \mathrm{fs}$ pulse. Because of the limit of the choice of a soliton ansatz for short pulses, there is a greater disagreement between the full simulation of Eq. (1) (dashed) to both

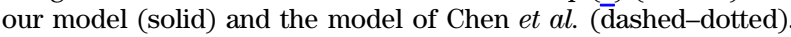

of agreement with the simulation of Eq. (1). For a pulse width of $10 \mathrm{fs}$, as shown in Fig. 2, the mismatch between the prediction of our model and that of Chen $e t a l$., with respect to the simulation of Eq. (1) becomes more significant. This is caused by TOD and self-steepening having a greater effect, thus causing the proposed soliton ansatz to be limiting the model.

Figure 2 also shows that although our model and that of Chen et $a \bar{l}$. give similar predictions, the agreement becomes poorer with decreasing pulse width, most noticeably in the different period of the fluctuations. By rewriting our model, it is possible to directly compare it to the model of Chen et al., as detailed in Appendix B, and it shows that there is a slight difference in four of the coupled equations between our model and that of Chen et al. For broad pulses, our model and that of Chen et al. reduce to the same set of differential equations, which explains the better agreement in Fig. 1(a). After careful scrutiny, no mistakes were found in this work, so the origin of the difference remains uncertain.

In general, a better agreement between our model and the full simulation of Eq. (1) is obtained for long pulses and less influence of TOD, as in these cases, the proposed ansatz function is a better match to the exact soliton solution.

\section{EFFECT OF TWO-PHOTON ABSORPTION}

To demonstrate the effect of TPA on the SSFS, we again assume the silica material parameters from the previous section with a 50 fs soliton; however, we now also assume a nonzero TPA coefficient for silica of $\mathrm{FOM}=5$ and $\mathrm{FOM}=1$. These values for the TPA coefficient at $1550 \mathrm{~nm}$ are not physical, as TPA is only relevant for silica at ultraviolet frequencies. For comparison, the TPA coefficient in silica at $355 \mathrm{~nm}$ is less than $1.25 \times 10^{-14} \mathrm{~m} / \mathrm{W}$ [37], 3 orders of magnitude smaller than the values listed in Table 1 for chalcogenide. Figure 3 shows the shift in wavelength for decreasing values of the FOM. The imaginary part of the nonlinear parameter expressed as a function of the FOM is $\gamma_{0}^{i}=\gamma_{0}^{r} /(4 \pi \cdot \mathrm{FOM})$.
Table 1. Used Values of the Nonlinearity and TwoPhoton Absorption for a $\mathrm{As}_{2} \mathrm{Se}_{3}$ Chalcogenide Fiber by Nguyen et al. [31]

\begin{tabular}{lccl}
\hline$\lambda(\mathrm{nm})$ & $n_{2}\left(10^{-18} \mathrm{~m}^{2} / \mathrm{W}\right)$ & $\beta_{\text {TPA }}\left(10^{-11} \mathrm{~m} / \mathrm{W}\right)$ & FOM \\
\hline 1415 & $11.0 \dagger^{a}$ & 0.97 & $0.8 \dagger$ \\
1434 & $14.0 \dagger$ & 1.10 & $0.9 \dagger$ \\
1456 & $13.0 \dagger$ & $0.80 \dagger$ & $1.1 \dagger$ \\
1491 & $9.9 \dagger$ & $0.46 \dagger$ & $1.45 \dagger$ \\
1515 & $8.9 \dagger$ & 0.31 & $1.9 \dagger$ \\
1554 & $7.0 \dagger$ & 0.25 & $1.8 \dagger$ \\
\hline
\end{tabular}

${ }^{a}$ The $\dagger$ symbol marks values read off from a figure.

The TPA is assumed constant over the wavelength range we look at and thus $\gamma_{1}^{i}=0$.

As would be expected, an increased value of the TPA coefficient leads to a decreased redshift, the primary reason being the loss of peak power for the soliton. Because the peak power is greater for shorter solitons, the effect of TPA will be more significant for shorter solitons. It also shows from Fig. 3 that there is a good qualitative agreement between our model and the simulation of the GNLS when TPA is included. The nonlinear loss length has been plotted as vertical lines, which shows that it is a good measure of the distance after which the power is sufficiently low as to effectively halt the redshift. Comparing our expression for $L_{\mathrm{Loss}}$, Eq. (9), with our model and the full simulation shows that the power is actually decreased below $1 / e$ of the initial value at the predicted distance $L_{\text {Loss }}$. This effect is due to our definition of $L_{\text {Loss }}$, in which we neglect the effects of pulse broadening, which also reduces the peak power.

\section{REDSHIFT IN CHALCOGENIDE FIBER}

Some soft glasses, like the chalcogenide materials arsenic selenide, $\mathrm{As}_{2} \mathrm{~S}_{3}$, and arsenic sulfide, $\mathrm{As}_{2} \mathrm{~S}_{3}$, have substantial TPA coinciding with the wavelengths used in telecommunication, which can limit the performance of these materials. Table 1 lists measured values of the nonlinear parameters for arsenic

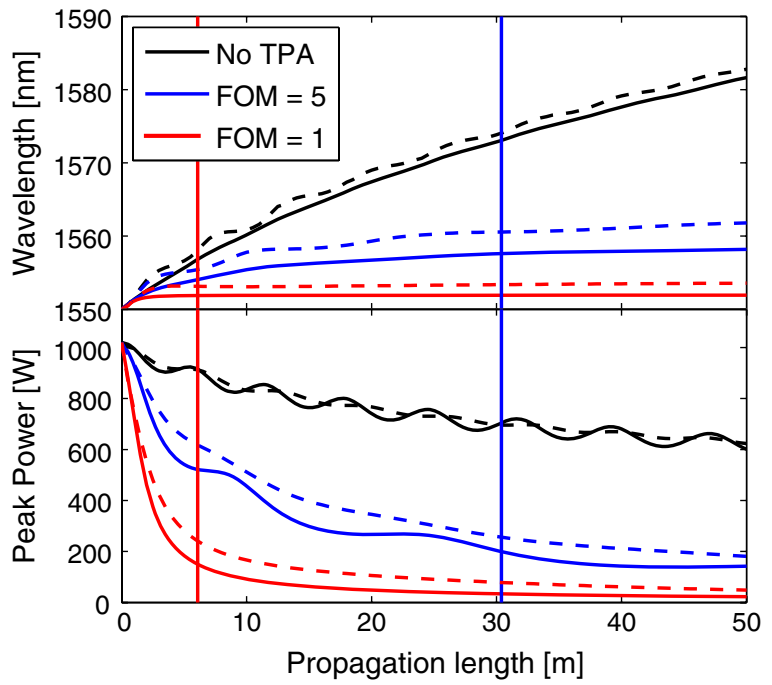

Fig. 3. (Color online) Center wavelength (top) and peak power (bottom) for our model (solid) and simulation of Eq. (1) (dashed) for a $T=50$ fs pulse with three different values of the TPA. The vertical lines in the lower plot mark the nonlinear loss length as given by Eq. (9). 

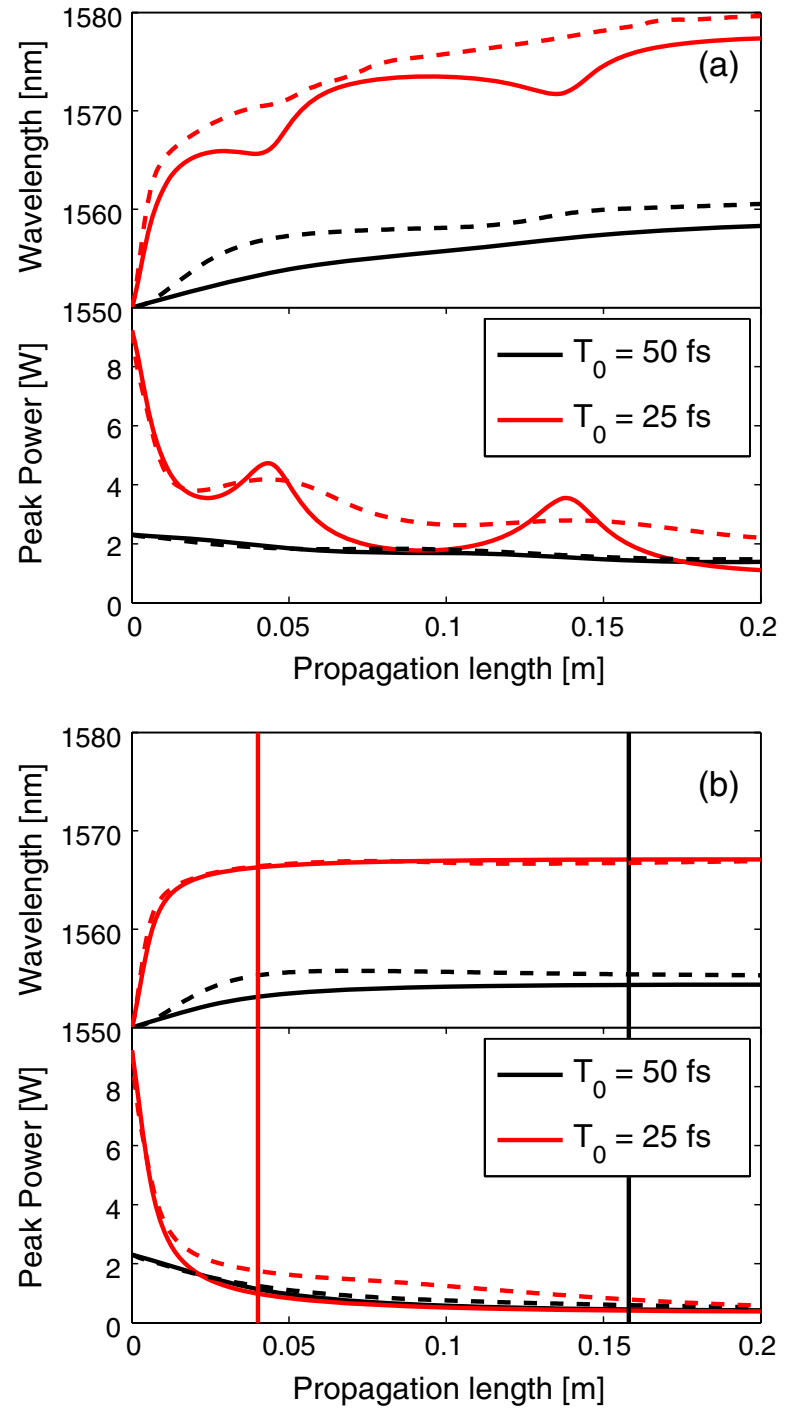

Fig. 4. (Color online) Evolution of center wavelength (top) and peak power (bottom) for a chalcogenide fiber for cases where TPA is (a) neglected and (b) included as found by our model (solid) and the simulation of Eq. (1) (dashed). The nonlinear loss length is marked by the vertical lines in (b).

selenide found in the literature. In Yeom et al. [15], a chalcogenide fiber was tapered in order to decrease the ZDW below $1550 \mathrm{~nm}$, enabling the possibility to pump the fiber in the anomalous dispersion regime with an erbium fiber laser to create a mid-infrared supercontinuum. The obtained dispersion and effective area are found to be $\beta_{2}=-360 \mathrm{ps}^{2} / \mathrm{km}$, $\beta_{3}=3.85 \mathrm{ps}^{3} / \mathrm{km}$ and $A_{\text {eff }}=0.4773 \mu \mathrm{m}^{2}$. The nonlinear coefficients are calculated from the values in Table 1 with the assumption that the effective area is independent of the frequency in order to differentiate $\gamma(\omega)$. Finally, the Raman response function, $h_{R}(t)$, is based on $\mathrm{Hu}$ et al. [38], where we, by fitting to the gain peak position and width, have approximated it as a decaying harmonic oscillator,

$$
h_{R}(t)=\frac{\tau_{1}^{2}+\tau_{2}^{2}}{\tau_{1} \tau_{2}^{2}} \exp \left(-\frac{t}{\tau_{2}}\right) \sin \left(\frac{t}{\tau_{1}}\right),
$$

with $\tau_{1}=23 \mathrm{fs}, \tau_{2}=210 \mathrm{fs}$, and $f_{R}=0.1$.
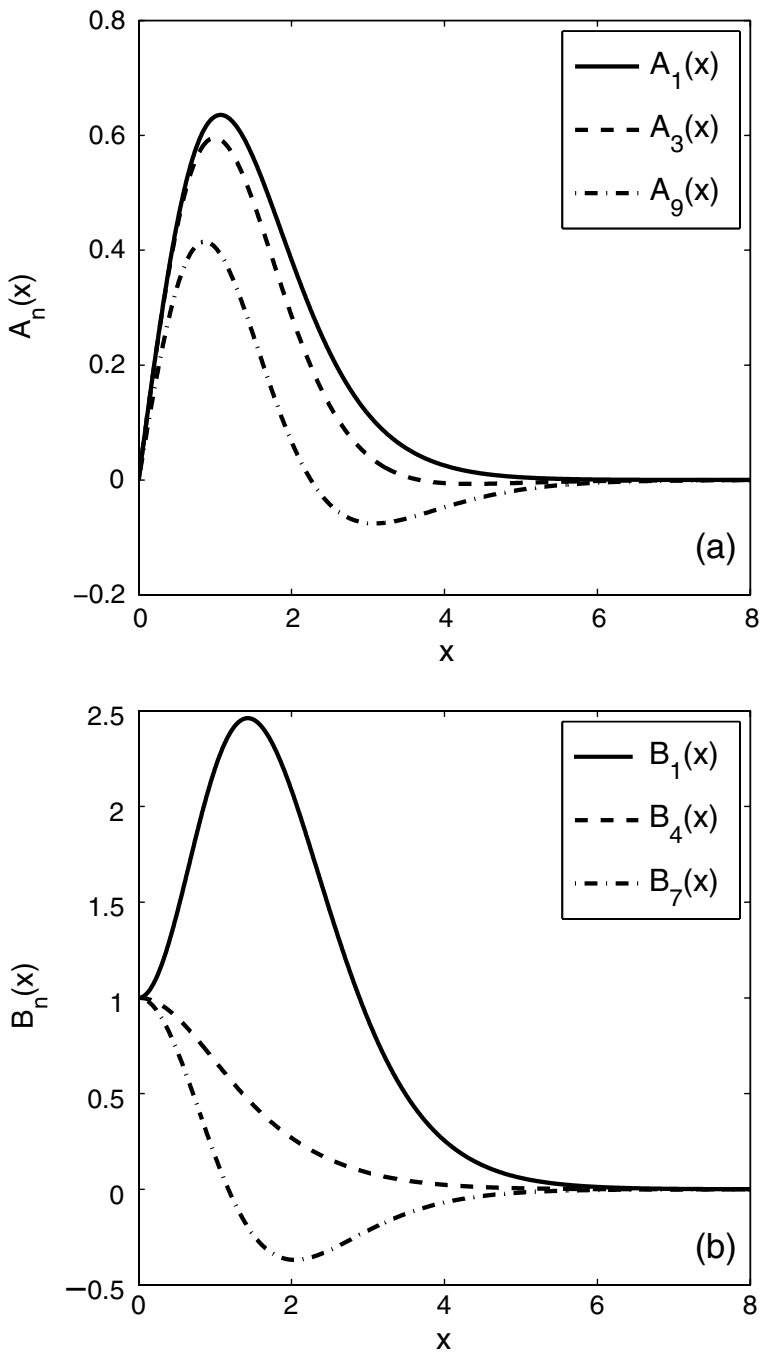

Fig. 5. (a) $A_{n}$ and (b) $B_{n}$ functions used in Eq. (B3).

Figure $\underline{4}$ shows the evolution in central wavelength and peak power for the case where TPA is accounted for and where it is neglected. As was also seen in Fig. 3, TPA can have a strong influence on the predicted wavelength shift, especially for shorter pulses as the peak power is greater thus suffering more from the TPA. Without TPA, one would predict a redshift of $27 \mathrm{~nm}$ over a $20 \mathrm{~cm}$ fiber for a $25 \mathrm{fs}$ pulse. However, we find that in reality the TPA will stop the redshift already at $L=4 \mathrm{~cm}$ and limit it to only $17 \mathrm{~nm}$.

\section{CONCLUSION}

The proposed model has been shown to describe SSFS, even for pulses as short as $25 \mathrm{fs}$, and has been shown to successfully account for the reduced SSFS under the influence of TPA. The model incorporates the full Raman spectrum and for the first time also TPA. We have shown the decrease in redshift due to TPA in a realistic chalcogenide waveguide used for SCG, which shows the necessity of including TPA when working with chalcogenide materials. Furthermore, we have defined a nonlinear loss length, which suits as a measure for the length after which SSFS is effectively halted, due to loss of power in waveguides where TPA is present. 

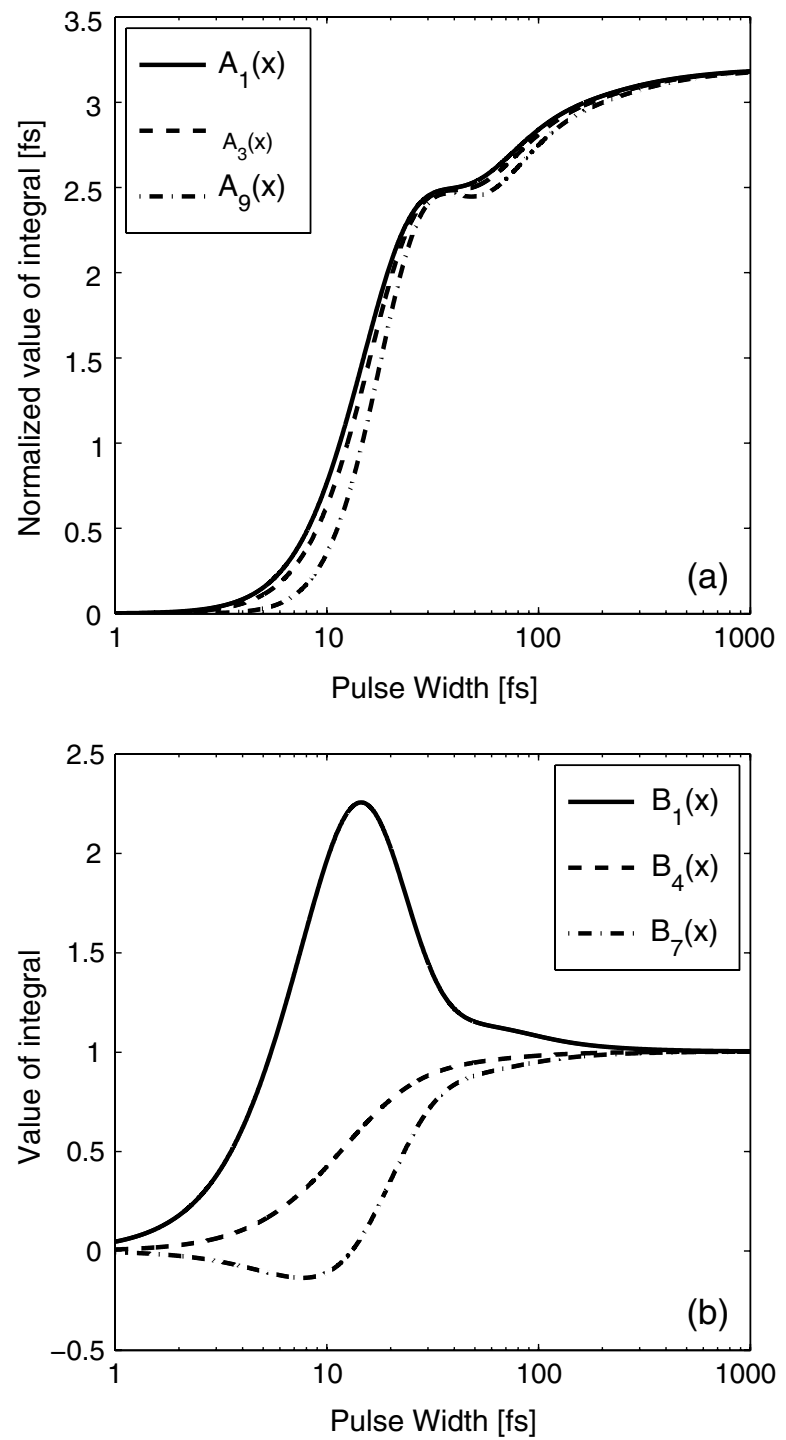

Fig. 6. Value of the integrals over (a) $A_{n}$ and (b) $B_{n}$ functions from Eq. (B3) as a function of pulse width. The integral over $A_{n}$ has been multiplied with $f_{R} T$ to show that the integral goes toward $T_{R} /\left(f_{R} T\right)$ when the pulse width is increased.

\section{APPENDIX A: COMPLETE SOLUTION}

$$
\begin{aligned}
\partial_{z} b= & -\frac{8}{15} \frac{\left(\gamma_{0}^{r}+b \gamma_{1}^{r}\right) P}{T} f_{R} \int h_{R}(t) A_{1}\left(\frac{t}{T}\right) \mathrm{d} t \\
& +\frac{4}{3} \mu \gamma_{1}^{r} P\left[1-f_{R}+f_{R} \int h_{R}(t) B_{1}\left(\frac{t}{T}\right) \mathrm{d} t\right] \\
& -\frac{4}{3} \mu\left(\gamma_{0}^{i}+b \gamma_{1}^{i}\right) P T f_{R} \int h_{R}(t) A_{2}\left(\frac{t}{T}\right) \mathrm{d} t \\
& -\frac{4}{5} \frac{\gamma_{1}^{i} P}{T^{2}}\left[1-f_{R}+f_{R} \int h_{R}(t) B_{2}\left(\frac{t}{T}\right) \mathrm{d} t\right] \\
& -4\left(\frac{\pi^{2}}{9}-\frac{2}{3}\right) \mu^{2} \gamma_{1}^{i} P T^{2}\left[1-f_{R}+f_{R} \int h_{R}(t) B_{3}\left(\frac{t}{T}\right) \mathrm{d} t\right],
\end{aligned}
$$

$$
\begin{aligned}
\partial_{z} t_{c}= & b\left(\beta_{2}+b \beta_{3}\right)+\frac{1}{2} \beta_{3}\left[\frac{\pi^{2}}{3} \mu^{2} T^{2}+\frac{1}{3 T^{2}}-b^{2}\right] \\
& +\gamma_{1}^{r} P\left[1-f_{R}+f_{R} \int h_{R}(t) B_{4}\left(\frac{t}{T}\right) \mathrm{d} t\right] \\
& -\frac{2}{3}\left(\gamma_{0}^{i}+b \gamma_{1}^{i}\right) P T f_{R} \int h_{R}(t) A_{2}\left(\frac{t}{T}\right) \mathrm{d} t \\
& -2\left(\frac{\pi^{2}}{9}-\frac{2}{3}\right) \mu \gamma_{1}^{i} P T^{2}\left[1-f_{R}+f_{R} \int h_{R}(t) B_{3}\left(\frac{t}{T}\right) \mathrm{d} t\right],
\end{aligned}
$$

$$
\begin{aligned}
\partial_{z} T= & 2 \mu T\left(\beta_{2}+b \beta_{3}\right)+\frac{8}{\pi^{2}} \gamma_{1}^{r} P f_{R} \int h_{R}(t) A_{3}\left(\frac{t}{T}\right) \mathrm{d} t \\
& +\frac{4}{\pi^{2}}\left(\gamma_{0}^{i}+b \gamma_{1}^{i}\right) P T\left[1-f_{R}+f_{R} \int h_{R}(t) B_{5}\left(\frac{t}{T}\right) \mathrm{d} t\right] \\
& -\left(\frac{4}{3}-\frac{12}{\pi^{2}}\right) \mu \gamma_{1}^{i} P T^{2} f_{R} \int h_{R}(t) A_{4}\left(\frac{t}{T}\right) \mathrm{d} t
\end{aligned}
$$

$$
\begin{aligned}
\partial_{z} P= & -\alpha P-2 \mu P\left(\beta_{2}+b \beta_{3}\right) \\
& -\left(\frac{8}{\pi^{2}}+\frac{8}{15}\right) \frac{\gamma_{1}^{r} P^{2}}{T} f_{R} \int h_{R}(t) A_{5}\left(\frac{t}{T}\right) \mathrm{d} t \\
& -\left(\frac{4}{3}+\frac{4}{\pi^{2}}\right)\left(\gamma_{0}^{i}+b \gamma_{1}^{i}\right) P^{2}\left[1-f_{R}+f_{R} \int h_{R}(t) B_{6}\left(\frac{t}{T}\right) \mathrm{d} t\right] \\
& -\frac{12}{\pi^{2}} \mu \gamma_{1}^{i} P^{2} T f_{R} \int h_{R}(t) A_{6}\left(\frac{t}{T}\right) \mathrm{d} t, \\
\partial_{z} \mu= & \left(\beta_{2}+b \beta_{3}\right)\left(\frac{2}{\pi^{2}} \frac{1}{T^{4}}-2 \mu^{2}\right) \\
& +\frac{2}{\pi^{2}} \frac{\left(\gamma_{0}^{r}+b \gamma_{1}^{r}\right) P}{T^{2}}\left[1-f_{R}+f_{R} \int h_{R}(t) B_{7}\left(\frac{t}{T}\right) \mathrm{d} t\right] \\
& -\left(\frac{8}{15}-\frac{4}{\pi^{2}}\right) \frac{\mu \gamma_{1}^{r} P}{T} f_{R} \int h_{R}(t) A_{7}\left(\frac{t}{T}\right) \mathrm{d} t \\
& -\frac{76}{15 \pi^{2}} \frac{\gamma_{1}^{i} P}{T^{3}} f_{R} \int h_{R}(t) A_{8}\left(\frac{t}{T}\right) \mathrm{d} t .
\end{aligned}
$$

The eight $A_{n}(x)$ functions,

$$
A_{1}(x)=\frac{15}{8} \operatorname{csch}^{4}(x)[4 x+2 x \cosh (2 x)-3 \sinh (2 x)]
$$

$$
A_{2}(x)=3 x \operatorname{csch}^{3}(x)[x \cosh (x)-\sinh (x)]
$$

$$
A_{3}(x)=\frac{1}{4} \operatorname{csch}^{4}(x)\left[-\left(2 x^{3}+6 x\right) \cosh (2 x)+9 x^{2} \sinh (2 x)\right.
$$$$
\left.-4 x^{3}+6 x\right]
$$

$$
\begin{aligned}
A_{4}(x)= & \frac{3}{\pi^{2}-9} x \operatorname{csch}^{3}(x)\left[\left(3 x^{3}+\pi^{2} x\right) \cosh (x)\right. \\
& \left.-\left(6 x^{2}+\pi^{2}\right) \sinh (x)\right],
\end{aligned}
$$

$$
\begin{aligned}
A_{5}(x)= & \frac{15}{8 \pi^{2}+120} \operatorname{csch}^{4}(x)\left[\left(18 x^{2}-3 \pi^{2}\right) \sinh (2 x)\right. \\
& \left.-\left(4 x^{3}+12 x-2 \pi^{2} x\right) \cosh (2 x)-8 x^{3}+12 x+4 \pi^{2} x\right],
\end{aligned}
$$




$$
\begin{gathered}
A_{6}(x)=\frac{1}{304} \operatorname{csch}^{5}(x)\left[\left(68 \pi^{2} x^{2}-180 x^{2}+75 \pi^{2}-450\right) \cosh (3 x)\right. \\
-\left(158 \pi^{2} x-720 x\right) \sinh (3 x)+\left(412 \pi^{2} x^{2}-2700 x^{2}-75 \pi^{2}\right. \\
\left.+450) \cosh (x)-\left(306 \pi^{2} x-2520 x\right) \sinh (x)\right], \\
A_{7}(x)=\frac{15}{8 \pi^{2}-60} \operatorname{csch}^{4}(x)\left[16 x^{3}+4 \pi^{2} x-6 x\right. \\
+\left(8 x^{3}+2 \pi^{2} x+6 x\right) \cosh (2 x) \\
\left.-\left(18 x^{2}+3 \pi^{2}\right) \sinh (2 x)\right],
\end{gathered}
$$

$$
\begin{aligned}
A_{8}(x)= & \frac{45}{152} \operatorname{csch}^{5}(x)[8 x \sinh (3 x)+28 x \sinh (x) \\
& \left.-\left(2 x^{2}+5\right) \cosh (3 x)-\left(30 x^{2}-5\right) \cosh (x)\right]
\end{aligned}
$$

have been normalized so $A_{n}(x \overrightarrow{0})=x$, while the seven $B_{n}(x)$ functions

$$
B_{1}(x)=\frac{3}{2} \operatorname{csch}^{4}(x)\left[\left(6 x^{2}-1\right) \cosh (2 x)-8 x \sinh (2 x)+12 x^{2}+1\right],
$$

$$
B_{2}(x)=\frac{5}{12} \operatorname{csch}^{5}(x)[7 \sinh (3 x)+27 \sinh (x)-3 x \cosh (3 x)
$$$$
-45 x \cosh (x)]
$$

$B_{3}(x)=\frac{3}{\pi^{2}-6} \operatorname{csch}^{3}(x)\left[\left(4 x^{3}+\pi^{2} x\right) \cosh (x)-\left(6 x^{2}+\pi^{2}\right) \sinh (x)\right]$,

$$
\begin{aligned}
B_{4}(x)= & \frac{1}{36} \operatorname{csch}^{5}(x)\left[\left(18 x^{2}+61\right) \sinh (3 x)\right. \\
& +\left(54 x^{2}+297\right) \sinh (x) \\
& -48 x \cosh (3 x)-432 x \cosh (x)]
\end{aligned}
$$

$$
B_{5}(x)=\operatorname{csch}^{3}(x)\left[3 x^{2} \sinh (x)-2 x^{3} \cosh (x)\right],
$$

$B_{6}(x)=\frac{3}{\pi^{2}+3} \operatorname{csch}^{3}(x)\left[\left(3 x^{2}-\pi^{2}\right) \sinh (x)-\left(2 x^{3}-\pi^{2} x\right) \cosh (x)\right]$,

$$
B_{7}(x)=\frac{3}{2} \operatorname{csch}^{4}(x)\left[4 x \sinh (2 x)-\left(2 x^{2}+1\right) \cosh (2 x)-4 x^{2}+1\right],
$$

are normalized so $B_{n}(x \overrightarrow{0})=1$. The normalization of these functions is such that all integrals over $B_{n}$ functions in Eq. (1ㅡ) go toward 1 as the pulse width is increased, while integrals over $A_{n}$ functions go toward $T_{R} /\left(f_{R} T\right)$. Reducing the complete solution to Eq. (5) is thus straightforward. Not all of these functions are linearly independent; it shows that

$$
A_{5}(x)=\frac{15}{\pi^{2}+15}\left[\frac{\pi^{2}}{15} A_{1}(x)+A_{3}(x)\right],
$$

$$
\begin{gathered}
A_{8}(x)=\frac{1}{\pi^{2}-6}\left[\pi^{2} A_{2}(x)-6 A_{6}(x)\right], \\
B_{6}(x)=\frac{1}{\pi^{2}+3}\left[9 B_{5}(x)+\left(\pi^{2}-6\right) B_{3}(x)\right], \\
B_{7}(x)=2 B_{2}(x)+\frac{2 \pi^{2}-12}{\pi^{2}} B_{3}(x)-3 B_{4}(x)+\frac{12}{\pi^{2}} B_{5}(x) .
\end{gathered}
$$

\section{APPENDIX B: SOLUTION WHEN NEGLECTING TPA AND COMPARISON WITH PREVIOUSLY PUBLISHED WORK}

The complete model is as given by Eq. (A1); here we show the reduced solution in the case where $\overline{\mathrm{TPA}}$ is neglected, thus $\gamma_{n}^{i}=0$

$$
\begin{aligned}
\partial_{z} b= & -\frac{8}{15} \frac{\left(\gamma_{0}^{r}+b \gamma_{1}^{r}\right) P}{T} f_{R} \int h_{R}(t) A_{1}\left(\frac{t}{T}\right) \mathrm{d} t \\
& +\frac{4}{3} \mu \gamma_{1}^{r} P\left[1-f_{R}+f_{R} \int h_{R}(t) B_{1}\left(\frac{t}{T}\right) \mathrm{d} t\right],
\end{aligned}
$$

$$
\begin{aligned}
\partial_{z} t_{c}= & b\left(\beta_{2}+b \beta_{3}\right)+\frac{1}{2} \beta_{3}\left[\frac{\pi^{2}}{3} \mu^{2} T^{2}+\frac{1}{3 T^{2}}-b^{2}\right] \\
& +\gamma_{1}^{r} P\left[1-f_{R}+f_{R} \int h_{R}(t) B_{4}\left(\frac{t}{T}\right) \mathrm{d} t\right]
\end{aligned}
$$

$$
\partial_{z} T=2 \mu T\left(\beta_{2}+b \beta_{3}\right)+\frac{8}{\pi^{2}} \gamma_{1}^{r} P f_{R} \int h_{R}(t) A_{3}\left(\frac{t}{T}\right) \mathrm{d} t
$$

$$
\begin{aligned}
\partial_{z} P= & -\alpha P-2 \mu P\left(\beta_{2}+b \beta_{3}\right) \\
& -\left(\frac{8}{\pi^{2}}+\frac{8}{15}\right) \frac{\gamma_{1}^{r} P^{2}}{T} f_{R} \int h_{R}(t) A_{5}\left(\frac{t}{T}\right) \mathrm{d} t,
\end{aligned}
$$

$$
\begin{aligned}
\partial_{z} \mu= & \left(\beta_{2}+b \beta_{3}\right)\left(\frac{2}{\pi^{2}} \frac{1}{T^{4}}-2 \mu^{2}\right) \\
& +\frac{2}{\pi^{2}} \frac{\left(\gamma_{0}^{r}+b \gamma_{1}^{r}\right) P}{T^{2}}\left[1-f_{R}+f_{R} \int h_{R}(t) B_{7}\left(\frac{t}{T}\right) \mathrm{d} t\right] \\
& -\left(\frac{8}{15}-\frac{4}{\pi^{2}}\right) \frac{\mu \gamma_{1}^{r} P}{T} f_{R} \int h_{R}(t) A_{7}\left(\frac{t}{T}\right) \mathrm{d} t .
\end{aligned}
$$

In order to ease the comparison to the work of Chen et al., the peak power is rewritten to pulse energy and the chirp is normalized according to

$$
E=\frac{2 P}{T}
$$




$$
C=\frac{\mu}{2 T^{2}}
$$

With this redefinition, the differential equations can be expressed as

$$
\begin{aligned}
& \partial_{z} b=-\frac{4}{15} \frac{\left(\gamma_{0}^{r}+b \gamma_{1}^{r}\right) E}{T^{2}} f_{R} \int h_{R}(t) A_{1}\left(\frac{t}{T}\right) \mathrm{d} t \\
& +\frac{1}{3} \frac{\gamma_{1}^{r} E C}{T^{3}}\left[1-f_{R}+f_{R} \int h_{R}(t) B_{1}\left(\frac{t}{T}\right) \mathrm{d} t\right], \\
& \partial_{z} t_{c}=b\left(\beta_{2}+b \beta_{3}\right)+\frac{1}{2} \beta_{3}\left[\frac{\pi^{2}}{12} \frac{C^{2}}{T^{2}}+\frac{1}{3 T^{2}}-b^{2}\right] \\
& +\frac{1}{2} \frac{\gamma_{1}^{r} E}{T}\left[1-f_{R}+f_{R} \int h_{R}(t) B_{4}\left(\frac{t}{T}\right) \mathrm{d} t\right] \\
& \partial_{z} T=\frac{\left(\beta_{2}+b \beta_{3}\right) C}{T}+\frac{4}{\pi^{2}} \frac{\gamma_{1}^{r} E}{T} f_{R} \int h_{R}(t) A_{3}\left(\frac{t}{T}\right) \mathrm{d} t, \\
& \partial_{z} E=-\alpha E-\frac{4}{15} \frac{\gamma_{1}^{r} E^{2}}{T^{2}} f_{R} \int h_{R}(t) A_{1}\left(\frac{t}{T}\right) \mathrm{d} t, \\
& \partial_{z} C=\left(C^{2}+\frac{4}{\pi^{2}}\right) \frac{\left(\beta_{2}+b \beta_{3}\right)}{T^{2}} \\
& +\frac{2}{\pi^{2}} \frac{\left(\gamma_{0}^{r}+b \gamma_{1}^{r}\right) E}{T}\left[1-f_{R}+f_{R} \int h_{R}(t) B_{7} \mathrm{~d} t\right] \\
& +\frac{150-4 \pi^{2}}{15 \pi^{2}} \frac{\gamma_{1}^{r} E C}{T^{2}} f_{R} \int h_{R}(t) A_{9}\left(\frac{t}{T}\right) \mathrm{d} t,
\end{aligned}
$$

where we have used Eq. (A4a) and introduced

$$
A_{9}(x)=\frac{1}{150-4 \pi^{2}}\left[120 A_{3}(x)-\left(4 \pi^{2}-30\right) A_{7}(x)\right],
$$

in order to ease the comparison. By direct comparison, it shows that a completely agreement with Chen et al. would require that

$$
\begin{gathered}
A_{3}(x)=A_{9}(x)=A_{1}(x) \\
=\frac{15}{8} \operatorname{csch}^{4}(x)[4 x+2 x \cosh (2 x)-3 \sinh (2 x)], \\
B_{1}(x)=B_{4}(x)=1 .
\end{gathered}
$$

Figure 5 shows the value of these functions, while Fig. 6 shows the value of the integral over these functions. It shows that while the $A_{n}$ functions differ, the integral value displays approximately the same value, whereas the integral over the $B_{n}$ functions shows a significant difference.

\section{REFERENCES}

1. B. T. Soifer and J. L. Pipher, "Instrumentation for infrared astronomy," Ann. Rev. Astron. Astrophys. 16, 335-369 (1978).

2. J. D. Monnie, "Optical interferometry in astronomy," Rep. Prog. Phys. 66, 789-857 (2003).

3. P. Rolfe, "In vivo near-infrared spectroscopy," Annu. Rev. Biomed. Eng. 02, 715-754 (2000).

4. B. Guo, Y. Wang, C. Peng, H. L. Zhang, G. P. Luo, H. Q. Le, C. Gmachl, D. L. Sivco, M. L. Peabody, and A. Y. Cho, "Laser-based mid-infrared reflectance imaging of biological tissues," Opt. Express 12, 208-219 (2004).

5. H. B. Gray, Chemical Bonds: An Introduction to Atomic and Molecular Structure (University Science, 1994).

6. P. Werle, F. Slemr, K. Maurer, R. Kormann, R. Mucke, and B. Janker, "Near- and mid-infrared laser-optical sensors for gas analysis," Opt. Lasers Eng. 37, 101-114 (2002).

7. Federation of American Scientists, "AN/AAQ-24 Directional Infrared Countermeasures (DIRCM)," http://www.fas.org/man/ dod-101/sys/ac/equip/an-aaq-24.htm.

8. J. M. Dudley, G. Genty, and S. Coen, "Supercontinuum generation in photonic crystal fiber," Rev. Mod. Phys. 78, 1135-1184 (2006).

9. N. Savage, "Supercontinuum sources," Nat. Photon. 3, 114-115 (2009).

10. C. Xia, M. Kumar, O. P. Kulkarni, M. N. Islam, F. L. Terry, M. J. Freeman, M. Poulain, and G. Maz, "Mid-infrared supercontinuum generation to $4.5 \mu \mathrm{m}$ in ZBLAN fluoride fibers by nanosecond diode pumping," Opt. Lett. 31, 2553-2555 (2006).

11. G. Qin, X. Yan, C. Kito, M. Liao, C. Chaudhari, T. Suzuki, and Y. Ohishi, "Ultrabroadband supercontinuum generation from ultraviolet to $6.28 \mu \mathrm{m}$ in a fluoride fiber," Appl. Phys. Lett. 95, 161103 (2009).

12. J. H. V. Price, T. M. Monro, H. Ebendorff-Heidepriem, F. Poletti, P Horak, V. Finazzi, J. Y. Y. Leong, P. Petropoulos, J. C. Flanagan, G Brambilla, X. Feng, and D. J. Richardson, "Mid-IR supercontinuum generation from nonsilica microstructured optical fibers," IEEE J. Sel. Top. Quantum Electron. 13, 738-749 (2007).

13. P. Domachuk, N. A. Wolchover, M. Cronin-Golomb, A. Wang, A. K. George, C. M. B. Cordeiro, J. C. Knight, and F. G. Omenetto, "Over $4000 \mathrm{~nm}$ bandwidth of mid-IR supercontinuum generation in sub-centimeter segments of highly nonlinear tellurite PCFs," Opt. Express 16, 7161-7168 (2008).

14. D. Buccoliero, H. Steffensen, O. Bang, H. Ebendorff-Heidepriem, and T. M. Monro, "Thulium pumped high power supercontinuum in loss-determined optimum lengths of tellurite photonic crystal fiber," Appl. Phys. Lett. 97, 061106 (2010).

15. D.-I. Yeom, E. C. Mägi, M. R. E. Lamont, M. A. F. Roelens, L. Fu, and B. J. Eggleton, "Low-threshold supercontinuum generation in highly nonlinear chalcogenide nanowires," Opt. Lett. 33, 660-662 (2008).

16. D. D. Hudson, S. A. Dekker, E. C. Mägi, A. C. Judge, S. D. Jackson, E. Li, J. S. Sanghera, L. B. Shaw, I. D. Aggarwal, and B. J. Eggleton, "Octave spanning supercontinuum in an $\mathrm{As}_{2} \mathrm{~S}_{3}$ taper using ultralow pump pulse energy," Opt. Lett. 36, 1122-1124 (2011).

17. T. M. Monro and H. Ebendorff-Heidepriem, "Progress in microstrusctured optical fibers," Ann. Rev. Mater. Res. 36, 467-495 (2006).

18. P. Russell, "Photonic crystal fibers," Science 299, 358-362 (2003).

19. W. Q. Zhang, S. Afshar V., and T. M. Monro, "A genetic algorithm based approach to fiber design for high coherence and large bandwidth supercontinuum generation," Opt. Express 17, 19311-19327 (2009).

20. T. A. Birks, W. J. Wadsworth, and P. St. J. Russell, "Supercontinuum generation in tapered fibers," Opt. Lett. 25, 1415-1417 (2000).

21. A. Kudlinski, A. K. George, J. C. Knight, J. C. Travers, A. B. Rulkov, S. V. Popov, and J. R. Taylor, "Zero-dispersion wavelength decreasing photonic crystal fibers for ultraviolet-extended supercontinuum generation," Opt. Express 14, 5715-5722 (2006).

22. S. T. Sørensen, A. Judge, C. L. Thomsen, and O. Bang, "Optimum tapers for increasing the power in the blue-edge of a supercontinuum-group-acceleration matching," Opt. Lett. 36, 816-818 (2011). 
23. C. Agger, S. T. Sørensen, C. L. Thomsen, S. R. Keiding, and O. Bang, "Nonlinear soliton matching between optical fibers," Opt. Lett. 36, 2596-2598 (2011).

24. J. P. Gordon, "Theory of the soliton self-frequency shift," Opt. Lett. 11, 662-664 (1986).

25. E. N. Tsoy and C. M. de Sterke, "Dynamics of ultrashort pulses near zero dispersion wavelength," J. Opt. Soc. Am. B 23, 2425-2433 (2006).

26. A. M. Zheltikov, "Perturbative analytical treatment of adiabatically moderated soliton self-frequency shift," Phys. Rev. E 75, 037603 (2007).

27. A. C. Judge, O. Bang, B. J. Eggleton, B. T. Kuhlmey, E. C. Mägi, R. Pant, and C. Martijn de Sterke, "Optimization of the soliton selffrequency shift in a tapered photonic crystal fiber," J. Opt. Soc. Am. B 26, 2064-2071 (2009).

28. A. C. Judge, O. Bang, and C. Martijn de Sterke, "Theory of dispersive wave frequency shift via trapping by a soliton in an axially nonuniform optical fiber," J. Opt. Soc. Am. B 27, 2195-2202 (2010).

29. Z. Chen, A. J. Taylor, and A. Efimov, "Soliton dynamics in nonuniform fiber tapers: analytical description through an improved moment method," J. Opt. Soc. Am. B 27, 1022-1030 (2010).

30. L. Yin, Q. Lin, and G. P. Agrawal, "Soliton fission and supercontinuum generation in silicon waveguides," Opt. Lett. 32, 391-393 (2007).
31. H. Nguyen, K. Finsterbusch, D. Moss, and B. Eggleton, "Dispersion in nonlinear figure of merit of $\mathrm{As}_{2} \mathrm{Se}_{3}$ chalcogenide fibre," Electron. Lett. 42, 571-572 (2006).

32. A. I. Maimistov, "Evolution of single waves close to solitons of Schrödinger nonlinear equation,” J. Exp. Theor. Phys. 77, 727-731 (1993) [Zh. Eksp. Teor. Fiz. 104, 3620-3629 (1993)].

33. W. Krolikowski, O. Bang, N. Nikolov, D. Neshev, J. Wyller, J Rasmussen, and D. Edmundson, "Modulational instability, solitons and beam propagation in nonlocal nonlinear media," J. Opt. B 6, S288-S294 (2004).

34. Q. Kong, Q. Wang, O. Bang, and W. Krolikowski, "Analytical theory for dark nonlocal solitons," Opt. Lett. 35, 2152-2154 (2010).

35. E. N. Tsoy, A. Ankiewicz, and N. Akhmediev, "Dynamical models for dissipative localized waves of the complex GinzburgLandau equation," Phys. Rev. E 73, 036621 (2006).

36. C. Petersen, S. Dupont, C. Agger, J. Thøgersen, O. Bang, and S. Keiding, "Stimulated Raman scattering in soft glass fluoride fibers," J. Opt. Soc. Am. B 28, 2310-2313 (2011).

37. P. Liu, W. L. Smith, H. Lotem, J. H. Bechtel, N. Bloembergen, and R. S. Adhav, "Absolute two-photon absorption coefficients at 355 and 266 nm," Phys. Rev. B 17, 4620-4632 (1978).

38. J. Hu, C. R. Menyuk, L. B. Shaw, J. S. Sanghera, and I. D. Aggarwal, "Maximizing the bandwidth of supercontinuum generation in $\mathrm{As}_{2} \mathrm{Se}_{3}$ chalcogenide fibers," Opt. Express 18, 6722-6739 (2010) 